\title{
Robotic versus laparoscopic surgery for colorectal cancer: a case-control study
}

\author{
Jan Grosek ${ }^{1,2}$, Jurij Ales Kosir ${ }^{1}$, Primoz Sever ${ }^{1}$, Vanja Erculj ${ }^{3}$, Ales Tomazic ${ }^{1,2}$ \\ ${ }^{1}$ Department of Abdominal Surgery, Ljubljana University Medical Center, Ljubljana, Slovenia \\ ${ }^{2}$ Medical Faculty, University of Ljubljana, Ljubljana, Slovenia \\ ${ }^{3}$ Faculty of Criminal Justice and Security, University of Maribor, Ljubljana, Slovenia
}

Radiol Oncol 2021; 55(4): 433-438.

Received 29 January 2020

Accepted 20 April 2021

Correspondence to: Prof. Aleš Tomažič, M.D., Ph.D., Department of Abdominal Surgery, Ljubljana University Medical Center, Zaloška cesta 7, SI-1000 Ljubljana, Slovenia; E-mail: ales.tomazic@kclj.si

Disclosure: No potential conflicts of interest were disclosed.

This is an open access article under the CC BY-NC-ND license (http://creativecommons.org/licenses/by-nc-nd/4.0/).

Background. Robotic resections represent a novel approach to treatment of colorectal cancer. The aim of our study was to critically assess the implementation of robotic colorectal surgical program at our institution and to compare it to the established laparoscopically assisted surgery.

Patients and methods. A retrospective case-control study was designed to compare outcomes of consecutively operated patients who underwent elective laparoscopic or robotic colorectal resections at a tertiary academic centre from 2019 to 2020. The associations between patient characteristics, type of operation, operation duration, conversions, duration of hospitalization, complications and number of harvested lymph nodes were assessed by using univariate logistic regression analysis.

Results. A total of 83 operations met inclusion criteria, 46 robotic and 37 laparoscopic resections, respectively. The groups were comparable regarding the patient and operative characteristics. The operative time was longer in the robotic group ( $p<0.001)$, with fewer conversions to open surgery $(p=0.004)$, with less patients in need of transfusions $(p=0.004)$ and lower reoperation rate $(p=0.026)$. There was no significant difference between the length of stay $(p$ $=0.17)$, the number of harvested lymph nodes $(p=0.24)$ and the overall complications $(p=0.58)$.

Conclusions. The short-term results of robotic colorectal resections were comparable to the laparoscopically assisted operations with fewer conversions to open surgery, fewer blood transfusions and lower reoperation rate in the robotic group.

Key words: robotic surgery; laparoscopic surgery; minimally invasive surgery; colorectal cancer

\section{Introduction}

Surgical resection is still the main treatment modality for resectable colorectal cancer. Advances in surgery have allowed the widespread use of minimally invasive surgical techniques, which are represented by laparoscopic and robotic approaches as opposed to open approach.

Laparoscopic colorectal resections (LCR) are safe and offer patients better short-term results as open surgery with less postoperative pain, faster recovery, shorter hospitalization and better cosme- sis. Furthermore, they are oncologically equivalent to open surgery, as evidenced by multiple randomized studies. ${ }^{1-3}$ There is even some evidence that LCR result in better median overall survival for patients with stage II colon cancer, older than 75 years, when compared to open surgery. ${ }^{4}$

Robotic surgical systems were designed to overcome the limitations of laparoscopic surgery, offering better visualization with three-dimensional magnified view and stable camera platform, stabilization of tremors and greater dexterity of movements. Moreover, they also improve the ergonom- 
ics, possibly reducing fatigue of the operating surgeon. ${ }^{5}$ Key drawbacks include loss of haptic control, longer operative time and above all, increased financial costs.

Shortly after the introduction of robotic platforms, surgeons have begun to utilize robotic surgery for management of colorectal diseases and the number of procedures performed annually has steadily increased. ${ }^{6}$ The evolution and usage of robotic platform is well illustrated by bibliometric data, as more and more manuscripts are being published each year, from feasibility studies to case series and reviews, and, finally, more and more multi-centre trials. The abundance of published research clearly shows, how robotic assisted surgery has gained acceptance not only in the field of colorectal surgery, but across many surgical specialities. ${ }^{7}$ However, most of the studies have not demonstrated a major advantage of robotic colorectal resections (RCR) in comparison to laparoscopic resections. ${ }^{8}$ Some studies have shown a benefit of the robotic approach with fewer conversions. ${ }^{9}$ The ROLARR study has also shown this for the difficult rectal resections involving obese men with low rectal cancers. ${ }^{10}$

To evaluate the implementation of robotic platform at a tertiary medical centre we designed a retrospective case-control study to compare outcomes of patients who underwent elective laparoscopic or robotic colorectal resections.

\section{Patients and methods}

\section{Patients}

A retrospective review of patients that underwent either robotic or laparoscopic surgery for colorectal carcinoma was performed. Patients in both groups were consecutively operated in a two-year period; in 2019 (laparoscopic group) and 2020 (robotic group). All the operations were performed by a two-member surgical team. The data source was a prospectively maintained database in a single academic institution with previous history of performing laparoscopic assisted surgeries for many years. ${ }^{11,12}$ Approval for the study was obtained from the Medical Ethics Committee of the Republic of Slovenia.

A total of 83 patients were identified and included in the study; of these, 46 underwent robotic and 37 laparoscopic resections, respectively. The inclusion criteria were as follows: histologically proven adenocarcinoma of colon or upper rectum $(>10 \mathrm{~cm}$ from the anal verge); no previous or concurrent malignancy at other site; no evidence of distant metastasis at the time of the surgery; minimally invasive (i.e., laparoscopic, or robotic) operation. Patients with low or middle rectal cancer and those presenting as acute emergent cases (i.e., perforation, obstruction) were excluded from the study.

Preoperative (age, sex, body mass index [BMI], American Society of Anaesthesiologists [ASA] score and tumour location), intraoperative (operative time, conversion rate) and postoperative complications according to the Clavien-Dindo (CD) classification, number of all harvested lymph nodes (LN) and number of positive LN, length of hospital stay and pathologic stage according to the $8^{\text {th }}$ edition of the American Joint Committee on Cancer [AJCC]-TNM classification, reoperation and mortality rates were reviewed. ${ }^{13,14}$ The primary outcomes were conversion rates and hospital length of stay. Secondary outcomes were operative time, postoperative morbidity, and number of harvested lymph nodes.

\section{Surgical technique}

All operations were performed by a two-member surgical team. Both surgeons were highly experienced in open and laparoscopic colorectal surgery and underwent thorough training with proctorship before starting the robotic colorectal program.

Early in 2020 we started a robotic abdominal program, focusing at first on colorectal resections, both for benign and malignant diseases. As safety and feasibility of robotic colorectal surgery (RCS) are well established, most of currently published data focuses on evaluating perioperative data, comparing it to its laparoscopic counterpart. ${ }^{15}$

Patients in both groups underwent identical, standard preoperative workup and preparation, according to our institutional practice. This included full colonoscopy (partial in case of obstructive carcinoma) and contrast-enhanced computed tomography (CT) of the chest and the abdomen. Full mechanical bowel preparation was employed as per standard for all left sided lesions, while enema alone was employed for all right sided and for completely obstructive left sided lesions as well, respectively. Preoperative intravenous antibiotics were given to cover intestinal flora.

Patients were secured on a special no-slip foam in a modified lithotomy position for left-sided colectomies and rectal resections, while for right colectomies legs were extended and secured by wrapping circumferentially with a roller bandage.

All RCR were performed by a single-docking, totally robotic technique using da Vinci Robotic 
Surgical System Xi (Intuitive Surgical System, Sunnyvale, CA, USA). All cases were operated with a dual console system. The robotic cart was docked on the side of the tumour, either on the left or right side of patients. Four $8 \mathrm{~mm}$ robotic ports were placed diagonally, lying on an imaginary linear line. Configuration of two-left handed instruments and one right-handed instrument was employed. Additional $12 \mathrm{~mm}$ port (Airseal ${ }^{\mathrm{R}}$, Applied Medical, USA) was inserted for the assistant at the patient site.

In both left and right sided colectomies primary vascular control with high-tie of the appropriate vessels was obtained at first. After that, a medial to lateral dissection was performed, respecting the avascular embryological planes. ${ }^{15}$ After resecting the bowel with tumour, intracorporeal anastomosis was fashioned: side-to-side anastomosis for right and left colectomies, and end-to-end for sigmoid or anterior rectal resections using circular stapling device and the double-stapling technique. Bowel was safely extracted through small Pfannenstiel incision, with wound-protector inserted for protection from faecal or tumour spillage.

For laparoscopic resection four or five trocar technique placed in a rhomboid fashion was used. Primary vascular control, followed by medial-tolateral dissection was used, like already described in robotic technique. The specimen was exteriorised through mini-median incision with wound-protector, and, after resecting the bowel with tumour, the anastomosis was performed. A combination of intracorporeal and extracorporeal anastomoses were utilized, as per surgeon's discretion. The former was performed as previously described for robotic operation. When extracorporeal anastomoses were chosen for right or left colectomies, they were hand sewn (side-to-side or end-to-side for right sidedanastomoses and end-to-end for left-sided anastomoses) under direct visualizations.

Postoperative complications were stratified according to the CD classification system. ${ }^{13}$ Accordingly severe morbidity was identified when at least CD grade III or more occurred. Anastomotic leak was considered along with all conditions with clinical or radiological features of anastomotic dehiscence. Hence, it was defined, as per the International study group definition. ${ }^{16}$ Conversion was defined as the unplanned change from laparoscopy to open procedure or from robotic surgery to either laparoscopic or open approach. Operative time was considered as the time from the first skin incision until the last scar was sutured.

Early and frequent mobility was encouraged, and venous thromboembolism prophylaxis was started approx. 12 hours after the operation. Nasogastric tube was removed prior to the end of the operation, while drainage tube and Foley catheter were removed on postoperative day one. Patients were offered clear liquids in the evening on the day of the operation. In the absence of nausea, vomiting or abdominal discomfort they were quickly advanced from liquid to regular diet.

\section{Statistical analysis}

Categorical variables were described by frequencies and percentages, normally distributed continuous variables by means and standard deviations, others by medians and interquartile ranges (IQR). Normality of the distribution of continuous variables per treatment group was assessed by ShapiroWilk test. The association between patient characteristics, operation duration, conversions, duration of hospitalization, complications, reoperation, and type of operation was assessed by using univariate logistic regression analysis. When there were zero cases present in any of the cells of the contingency table, likelihood ratio test was used. All statistical testing was performed at 0.05 significance level. Statistical program SPSS version 27 was used to perform all statistical analyses.

\section{Results}

There was no statistically significant association between demographic variables, concomitant diseases, the severity of disease and the type of the operation (Table 1). Patient characteristics did not differ significantly between groups as there was no statistically significant association between demographic variables, concomitant diseases, and severity of disease and the type of the operation.

Associations between several variables of the performed operation, patient course of recovery after the operation, procedure (type of resection) and the type of the operation performed (laparoscopic or robotic) were analysed by univariate logistic regression and results are presented in Table 2. Operative time was statistically significantly longer $(p<0.001)$ in robotic group. Five $(13.5 \%)$ patients had operation conversion within the laparoscopic group, while there were no conversions to open surgery in the robotic group of patients $(p=0.004)$. While the groups were comparable regarding the duration of hospitalization $(p=0.168)$ and the number of harvested lymph nodes $(p=0.240)$, the transfusion was to higher extent given to patients 
TABLE 1. Association between demographic characteristics, concomitant diseases, severity of the disease, and type of operation (results of univariate logistic regression)

\begin{tabular}{|c|c|c|c|c|}
\hline & Laparoscopic $(n=37)$ & Robotic $(n=46)$ & OR $(95 \% \mathrm{Cl})$ & $\mathbf{P}$ \\
\hline Male gender & $23(62.2)$ & $26(56.5)$ & $0.79(0.33 ; 1.92)$ & 0.604 \\
\hline Mean age (SD) & $67.5(10.1)$ & $66.8(11)$ & $0.99(0.95 ; 1.04)$ & 0.770 \\
\hline Median (IQR) BMI & $27.2(25.1-29.4)$ & $27.5(25.7-31.3)$ & $1.01(0.92 ; 1.12)$ & 0.808 \\
\hline ASA & & & & $0.262^{a}$ \\
\hline 1 & $0(0)$ & $2(4.3)$ & & \\
\hline 2 & $20(54.1)$ & $24(52.2)$ & & \\
\hline 3 & $16(43.2)$ & $20(43.5)$ & & \\
\hline 4 & $1(2.7)$ & $0(0)$ & & \\
\hline Procedure & & & & 0.273 \\
\hline Right colectomy & $15(40.5)$ & $21(45.7)$ & - & - \\
\hline Left colectomy & $4(10.8)$ & $3(6.5)$ & - & - \\
\hline Rectosigmoid/sigmoid/anterior resection & $16(43.2)$ & $22(47.8)$ & - & - \\
\hline Total colectomy & $2(5.4)$ & $0(0)$ & - & - \\
\hline $\begin{array}{c}\text { T stage } \\
\mathrm{Tl}\end{array}$ & $9(24.3)$ & $7(15.2)$ & - & - \\
\hline T2 & 7 (18.9) & $13(28.3)$ & $2.39(0.69 ; 9.2)$ & 0.206 \\
\hline T3 & $15(40.5)$ & $20(43.5)$ & $1.71(0.52 ; 5.65)$ & 0.376 \\
\hline T4 & $6(16.2)$ & $6(13)$ & $1.29(0.29 ; 5.77)$ & 0.743 \\
\hline \multicolumn{5}{|l|}{ Stage } \\
\hline 1 & $16(43.2)$ & $12(26.1)$ & 1 & - \\
\hline 2 & $9(24.3)$ & $18(39.1)$ & $2.67(0.89 ; 7.98)$ & 0.079 \\
\hline 3 & $12(32.4)$ & $16(34.8)$ & $1.78(0.62 ; 5.12)$ & 0.287 \\
\hline
\end{tabular}

${ }^{a}=$ likelihood ratio test; $A S A=$ American Society of Anaesthesiologists score; $B M I=$ body mass index; $C l=$ confidence interval; $I Q R=$ interquartile range: $\mathrm{OR}=$ odds ratio; $\mathrm{SD}=$ standard deviation

in laparoscopic group of patients $(\mathrm{p}=0.004)$. Five (13.5\%) patients within laparoscopic group needed transfusion, while there were no patients in need of transfusion within the robotic group of patients. The groups were comparable both with regards to overall complications $(p=0.576)$ as well as to type of complications according to the CD classification $(p=0.12)$. Reoperation was performed in three $(8.1 \%)$ patients from the laparoscopic and none of the patients within the robotic group of patients $(\mathrm{p}$ $=0.026$ ).

\section{Discussion}

This analysis of minimally invasive colorectal resections is, to the best of our knowledge, the first comparison of robotic versus laparoscopic resections for colon and upper rectal cancer in Slovenia. Safety of our patients and quality of surgical care was of outmost importance when we implemented a new robotic abdominal program. Hence, treatment results were not to be compromised. Consequently, only patients with colon and upper rectal cancer were operated at first, because we deemed middle and low rectal cancers not suitable at the beginning of the new program, due to technical demands of pelvic surgery.

Many studies, comparing RCR and LCR, have shown the new technology to be safe, feasible and at least equivalent with respect to short-term outcomes and oncological results. ${ }^{17}$ The most relevant finding of our study were significantly lower rates of conversion to open with the robotic platform compared to LCR $(p=0.004)$, which is consistent with findings of other studies. ${ }^{18}$ Only slightly shorter hospital length of stay was seen in robotic group, the difference not reaching statistical significance $(p=0.168)$. Some studies agree with our findings, others, on the other hand, demonstrate 
TABLE 2. Association between the operation and hospitalization duration, conversion, number of lymph nodes, transfusion, complications, reoperation, and the type of the operation (results of univariate logistic regression)

\begin{tabular}{|c|c|c|c|c|}
\hline & Laparoscopic $(n=37)$ & Robotic $(n=46)$ & OR $(95 \% \mathrm{Cl})$ & $\mathbf{P}$ \\
\hline Median (IQR) operation duration (min) & $150(130-184)$ & $262(201-300)$ & $1.03(1.02 ; 1.05)$ & $\begin{array}{c}< \\
0.001\end{array}$ \\
\hline Conversion & $5(13.5)$ & $0(0)$ & & $0.004^{a}$ \\
\hline Median (IQR) hospitalization duration & $7(6-8)$ & $6(5-7)$ & $0.91(0.81 ; 1.04)$ & 0.168 \\
\hline Lymph nodes & $20(15-26)$ & $24(21-30)$ & $1.03(0.98 ; 1.08)$ & 0.24 \\
\hline Transfusion & $5(13.5)$ & $0(0)$ & & $0.004^{a}$ \\
\hline Complications & $10(27)$ & $10(21.7)$ & $0.75(0.27 ; 2.06)$ & 0.576 \\
\hline Clavien-Dindo & & & & $0.12^{\mathrm{a}}$ \\
\hline 0 & $27(73)$ & $36(78.3)$ & & \\
\hline 1 & $0(0)$ & $3(6.5)$ & & \\
\hline 2 & $7(18.9)$ & $6(13)$ & & \\
\hline 3 & $2(5.4)$ & $0(0)$ & & \\
\hline 5 & $1(2.7)$ & $1(2.2)$ & & \\
\hline Reoperation & $3(8.1)$ & $0(0)$ & & $0.026^{a}$ \\
\hline
\end{tabular}

$a=$ likelihood ratio test; $\mathrm{Cl}=$ confidence interval; $I Q R=$ interquartile range; $O R=$ odds ratio

clinically significant shorter length of stay associated with robotic colorectal resections. ${ }^{19-21}$ In our study, there were three cases of anastomotic leakage in laparoscopic group. All three patients had to be reoperated, one of them died due to the septic shock. In the robotic group, there was no anastomotic leakage, however, one patient died because of acute thrombosis of superior mesenteric artery and coeliac axis. It is reasonable to assume that our study was underpowered to detect differences in postoperative morbidity, either overall or specific complications. However, other studies also demonstrate inconsistencies when comparing laparoscopic and robotic complication rates. Halabi et al., in their analysis argue, that there is no difference in postoperative morbidity between LCR and RCR. ${ }^{22}$ On the other hand, there are studies, showing better results in terms of postoperative morbidity and mortality for either laparoscopic or robotic colorectal resections. ${ }^{23,24}$ Nevertheless, some reports in literature show variable differences in complication rates, i.e., more anastomotic complications in one group and more general postoperative morbidity in the other. ${ }^{20}$

Another limitation of our study is its retrospective observational nature with all the inherent biases. Important issue to be addressed are the operative costs, which is one of the biggest criticisms of robotic surgery and has been a subject of discussion since its introduction. Our database does not include the cost data, so we did not address this issue in our study. Also, the intention of study being primarily the safety and oncologic equivalency of our new robotic surgical program, we did not assess the quality of life of operated patients. This, together with comprehensive cost analysis as well as long-term oncological results represents a very good potential for future studies.

The strength of our study is that the operations were performed by only two surgeons that reduces the heterogeneity of surgical techniques. Both surgeons had years of experience in laparoscopic surgery for all the patients included in the study and it can be assumed that they were already on top of their learning curve in laparoscopic surgery. This probably also played a role in faster acquisition of skills on the robotic platform. However, since the included robotic operations represent the start of our robotic program, there may still be room for improvement. It has been shown that the operating time decreases with the number of cases and this could have impacted the results of our study as well. ${ }^{25,26}$

\section{Conclusions}

With this study, we sought to offer an outcomebased assessment of implemented robotic colorectal program at our academic institution. Based on 
the results, it is appropriate to conclude, that our program is safe, has equivalent postoperative results compared to classic laparoscopy and is even associated with decreased conversion rates.

\section{References}

1. Weeks JC, Nelson H, Gelber S, Sargent D, Schroeder G. Clinical outcomes of surgical therapy (COST) study group. Short-term quality-of-life outcomes following laparoscopic-assisted colectomy vs open colectomy for colon cancer: a randomized trial. JAMA 2020; 287: 321-8. doi: 10.1001/jama.287.3.321

2. Green BL, Marshall HC, Collinson F, Quirke P, Guillou P, Jayne DG, et al. Longterm follow-up of the medical research council CLASICC trial of conventional versus laparoscopically assisted resection in colorectal cancer. $\mathrm{Br} J$ Surg 2013; 100: 75-82. doi: 10.1002/bjs.8945

3. Deijen CL, Vasmel JE, de Lange-de Klerk ESM, Cuesta MA, Coene PLO, Lange $\mathrm{JF}$, et al. Ten-year outcomes of a randomised trial of laparoscopic versus open surgery for colon cancer. Surg Endosc 2017; 31: 2607-15. doi: 10.1007/ s00464-016-5270-6

4. Fan CZ, Chu YP, Wei P, Dai H, Chen W. Comparison of survival of patients receiving laparoscopic and open radical resection for stage II colon cancer Radiol Oncol 2011; 45: 273-8. doi: 10.2478/v10019-011-0029-0

5. Wee IJY, Kuo $\sqcup$, NGU JCU. A systematic review of the true benefits of robotic surgery: ergonomics. Int J Med Robotics Comput Assist Surg 2020; 16: e2113. doi: $10.1002 /$ rcs.2113

6. Sheetz KH, Claflin J, Dimick JB. Trends in the adoption of robotic surgery for common surgical procedures. JAMA Netw Open 2020; 3: e1018911. doi: 10.1001/jamanetworkopen.2019.18911

7. Connelly TM, Malik Z, Sehgal R, Byrnes G, Coffey JC, Peirce C. The 100 most influential manuscripts in robotic surgery: a bibliometric analysis. J Robot Surg 2020; 14: 155-65. doi: 10.1007/s11701-019-00956-9

8. Delaney CP, Lynch AC, Senagore AJ, Fazio VW. Comparison of robotically performed and traditional laparoscopic colorectal surgery. Dis Colon Rectum 2003; 46: 1633-9. doi: 10.1007/BF02660768

9. Speicher PJ, Englum BR, Ganapathi AM, Nussbaum DP, Mantyh CR, Migaly J. Robotic low anterior resection for rectal cancer: a national perspective on short-term oncologic outcomes. Ann Surg 2015; 262: 1040-5. doi: 10.1097/ SLA. 0000000000001017

10. Jayne D, Pigazzi A, Marshall H, Croft J, Corrigan N, Copeland J, et al. Effect of robotic-assisted vs conventional laparoscopic surgery on risk of conversion to open laparotomy among patients undergoing resection for rectal cancer: The ROLARR randomized clinical trial. JAMA 2017; 318: 1569-80. doi: 10.1001/jama.2017.7219

11. Grosek J, Tomazic A. Key clinical appliacations for indocyanine green fluorescence imaging in minimally invasive colorectal surgery. J Minim Access Surg 2020; 16: 308-14. doi: 10.4103/jmas.JMAS_312_18

12. Janež J, Korać T, Kodre AR, Jelenc F, Ihan A. Laparoscopically assisted colorectal surgery provides better short-term clinical and inflammatory outcomes compared to open colorectal surgery. Arch Med Sci 2015; 11: 1217-26. doi: 10.5114/aoms.2015.56348

13. Dindo D, Demartines N, Clavien PA. Classification of surgical complications: a new proposal with evaluation in a cohort of 6336 patients and results of a survey. Ann Surg 2004; 240: 205-13. doi: 10.1097/01. sla.0000133083.54934.ae

14. Weiser MR. AJCC $8^{\text {th }}$ edition: Colorectal cancer. Ann Surg Oncol 2018; 25 1454-5. doi: 10.1245/s10434-018-6462-1

15. Grosek J. How I do it: right colectomy with the Da Vinci Xi Robotic Platform. Surgery Surg Endos 2020; 2: 61-8.

16. Rahbari NN, Weitz J, Hohenberger W, Heald RJ, Moran B, Ulrich A, et al. Definition and grading of anastomotic leakage following anterior resection of the rectum: a proposal by the International study group of rectal cancer. Surgery 2010; 147: 339-51. doi: 10.1016/j.surg.2009.10.012
17. Ng KT, Tsia AKV, Chong VYL. Robotic versus conventional laparoscopic surgery for colorectal cancer: a systematic review and meta-analysis with trial sequential analysis. World J Surg 2019; 43: 1146-61. doi: 10.1007/ s00268-018-04896-7

18. Liao G, Zhao Z, Lin S, Li R, Yuan Y, Du S, et al. Robotic-assisted versus laparoscopic colorectal surgery: a meta-analysis of four randomized controlled studies. World J Surg 2014; 12: 1-11. doi: 10.1186/1477-7819-12-122

19. Rawlings AL, Woodland JH, Vegunta RK, Crawford DL. Robotic versus laparoscopic colectomy. Surg Endosc 2007; 21: 1701-8. doi: 10.1007/ s00464-007-9231-y

20. Tam MS, Kaoutzanis C, Mullard AJ, Regen SE, Franz MG, et al. A populationbased study comparing laparoscopic and robotic outcomes in colorectal surgery. Surg Endosc 2016; 30: 455-63. doi: 10.1007/s00464-015-4218-6

21. Kim CW, Kim CH, Baik SH. Outcomes of robotic-assisted colorectal surgery compared with laparoscopic and open surgery: a systematic review. J Gastrointest Surg 2014; 18: 816-30. doi: 10.1007/s11605-014-2469-5

22. Halabi WJ, Kang CY, Jafari MD, Nguyen VQ, Carmichael JC, Mills S, et al. Robotic-assisted colorectal surgery in the United States: a nationwide analysis of trend and outcomes. World J Surg 2013; 37: 2782-90. doi: 10.1007/ s00268-013-2024-7

23. Salman M, Bell T, Martin J, Bhuva K, Grim R, Ahuja V. Use, cost, complications and mortality of robotic versus nonrobotic general surgery procedures based on a nationwide database. Ann Surg 2013; 79: 553-60

24. Tyler JA, Fox JP, Desai MM, Perry WB, Glasgow SC. Outcome and cost associated with robotic colectomy in the minimally invasive era. Dis Colon Rectum 2013; 56: 458-66. doi: 10.1097/DCR.0b013e31827085ec

25. Mushtaq HH, Shah SK, Agarwal AK. The current role of robotics in colorectal surgery. Curr Gastroenterol Rep 2019; 21: 11. doi: 10.1007/s11894-0190676-7.

26. Shaw DD, Wright $M$, Taylor L, Bertelson NL, Shashidharan $M$, Menon $P$, et al. Robotic Colorectal Surgery Learning Curve and Case Complexity. $J$ Laparoendosc Adv Surg Tech A 2018; 28: 1163-8. doi: 10.1089/lap.2016.0411 